\title{
Association of the APOA1 rs964184 SNP and serum lipid traits in the Chinese Maonan and Han populations
}

\author{
Ling Qiu', Rui-Xing Yin ${ }^{1 *} \mathbb{D}$, Eksavang Khounphinith ${ }^{1}$, Fen-Han Zhang ${ }^{1}$, De-Zhai Yang ${ }^{2}$ and Shang-Ling Pan ${ }^{3}$
}

\begin{abstract}
Background: Maonan nationality belongs to a mountain ethnic minority in China. Little is known about the association of apolipoprotein A1 gene (APOA1) rs964184 single nucleotide polymorphism (SNP) and serum lipid levels in this population. The aim of this study was to detect the association of the APOA1 rs964184 SNP and several environmental factors with serum lipid profiles in the Chinese Maonan and Han populations.

Methods: Genotypes of the APOA1 rs964184 SNP in 867 individuals of Maonan nationality and 820 participants of Han nationality were determined by polymerase chain reaction and restriction fragment length polymorphism, combined with gel electrophoresis, and confirmed by direct sequencing.

Results: The frequencies of CC, CG and GG genotypes of the APOA1 rs964184 SNP were 68.86, 29.18 and 1.96\% in the Maonan population, and $63.78,30.85$ and $5.37 \%$ in the Han population $(P<0.001)$. The frequency of the $\mathrm{G}$ allele was 16.55\% in Maonan and 20.79\% in Han $(P<0.001)$. The G allele carriers had lower high-density lipoprotein cholesterol (HDL-C) levels in Maonan and higher triglyceride (TG) levels in Han peoples than the $\mathrm{G}$ allele non-carriers. Subgroup analyses showed that the $\mathrm{G}$ allele carriers had lower HDL-C levels in both Maonan males and females; and lower apolipoprotein (Apo) A1 levels and the ApoA1/ApoB ratio in Han males than the G allele non-carriers. Serum lipid parameters in the two ethnic groups were also associated with several environmental factors.
\end{abstract}

Conclusions: The present study reveals that there may be a racial/ethnic- and/or gender-specific association between the APOA1 rs964184 SNP and serum lipid parameters in our study populations.

Trial registration: Retrospectively registered.

Keywords: Lipids, Apolipoprotein A1, Single nucleotide polymorphism, Environmental factors

\section{Background}

Nowadays cardiovascular disease (CVD) becomes the primary cause of disability among adults found in some studies, and coronary artery disease (CAD) continues to be the leading cause of death and years of life lost around the world [1,2]. The number of deaths from CAD has increased 35\% over the 20 years from 1990 to 2010 [2]. It is widely acknowledged that dyslipidemia is one of the major risk factors for CVD from its prediction to development [3]. Dyslipidemia highly relates to

\footnotetext{
*Correspondence: yinruixing@163.com

${ }^{1}$ Department of Cardiology, Institute of Cardiovascular Diseases, The First Affiliated Hospital, Guangxi Medical University, 22 Shuangyong Road, Nanning 530021, Guangxi, People's Republic of China

Full list of author information is available at the end of the article
}

the risk of CAD, which related to increased serum levels of total cholesterol (TC) [4], triglyceride (TG) [5], lowdensity lipoprotein cholesterol (LDL-C) [6], and apolipoprotein (Apo) B [7], combined with decreased levels of high-density lipoprotein cholesterol (HDL-C) [8], ApoA1 and the ApoA1/ApoB ratio [8]. Several previous studies have demonstrated that low level of HDL-C is an independent risk factor of CAD $[9,10]$.

It is well established that abnormal serum lipid levels are concerned with genetic and multiple environmental factors and their interactions [11]. ApoA1 gene (APOA1) encodes a protein, ApoA1, in humans, which has a specific role in lipid metabolism [12, 13]. There is another study showed that the pathway of HDL-C metabolism involves Apo, and ApoA1 is the most abundant component

(c) The Author(s). 2018 Open Access This article is distributed under the terms of the Creative Commons Attribution 4.0 International License (http://creativecommons.org/licenses/by/4.0/), which permits unrestricted use, distribution, and 
of HDL-C [14]. APOA1 plays an essential role in formation, maturation and metabolism of HDL-C [15]. ApoA1 is also a better biomarker of cardiovascular risk [10]. Genome-wide association study (GWAS) has identified that genetic variant of the APOA1 rs964184 SNP was associated with serum TG, TC, HDL-C and LDL-C levels [16]. However, it has not been verified that the effect of the APOA1 rs964184 SNP on serum lipid levels, both in the mechanism and function. To the best of our knowledge, the association of the APOA1 rs964184 SNP and serum lipid levels has not been previously reported in the Maonan population. Therefore, this study was undertaken to assess the association of the APOA1 rs964184 SNP and several environmental factors with serum lipid profiles in the Maonan and Han populations.

\section{Methods}

\section{Subjects}

A total of 867 unrelated participants (329 males, 37.95\% and 538 females, 62.5\%) of Maonan nationality and 820 unrelated subjects (306 males, 37.32\% and 514 females, $62.68 \%$ ) of Han nationality were randomly selected from our previous stratified randomized samples [17]. The participants were all agricultural workers from Huanjiang Maonan Autonomous County, Guangxi Zhuang Autonomous Region, People's Republic of China. These participants' age ranged from 22 to 92 years with the mean age of $57.71 \pm 14.74$ years in Maonan and $56.34 \pm$ 15.63 years in Han, respectively. The age distribution and gender ratio were matched between the two ethnic groups. All participants were essentially healthy with no evidence of CVD such as CAD, stroke, diabetes, hyperor hypo-thyroids, and chronic renal disease. They were free from any treatment which would affect serum lipid levels. This study design was approved by the Ethics Committee of the First Affiliated Hospital, Guangxi Medical University (No. Lunshen-2014-KY-Guoji-001, Mar. 7, 2014). Informed consent was obtained from all participants before study.

\section{Epidemiological survey}

The epidemiological survey was performed using internationally standardized methods, following a common protocol [18]. Information on demographics, socioeconomic status, and lifestyle factors was collected with standardized questionnaires. The intake of alcohol was quantified as the number of liang (about $50 \mathrm{~g}$ ) of rice wine, corn wine, rum, beer, or liquor consumed during the preceding 12 months. Alcohol consumption was categorized into groups of grams of alcohol per day: 0 (non-drinker), $\leq 25$ and $>25$. Smoking status was categorized into groups of cigarettes per day: 0 (non-smoker), $\leq 20$ and $>20$. Several parameters such as height, weight, blood pressure, and waist circumference were measured.
Body mass index (BMI) was calculated as weight $/$ height $^{2}$ $\left(\mathrm{kg} / \mathrm{m}^{2}\right)$.

\section{Biochemical measurements}

A fasting venous blood sample of $5 \mathrm{ml}$ was drawn from the participants. A part of the sample $(2 \mathrm{~mL})$ was collected into glass tubes and used to determine serum lipid levels. Another part of the sample $(3 \mathrm{~mL})$ was transferred to tubes with anticoagulants $(4.80 \mathrm{~g} / \mathrm{L}$ citric acid, $14.70 \mathrm{~g} / \mathrm{L}$ glucose and $13.20 \mathrm{~g} / \mathrm{L}$ tri-sodium citrate) and used to extract deoxyribonucleic acid (DNA). Measurements of serum TC, TG, HDL-C, and LDL-C levels in the samples were performed by enzymatic methods with commercially available kits (RANDOX Laboratories Ltd., Ardmore, Diamond Road, and Crumlin Co. Antrim, United Kingdom, BT29 4QY; Daiichi Pure Chemicals Co., Ltd., Tokyo, Japan). Serum ApoA1 and ApoB levels were detected by the immunoturbidimetric immunoassay using a commercial kit (RANDOX Laboratories Ltd.). All determinations were performed with an autoanalyzer (Type 7170A; Hitachi Ltd., Tokyo, Japan) in the Clinical Science Experiment Center of the First Affiliated Hospital, Guangxi Medical University [19].

\section{DNA amplification and genotyping}

Genomic DNA of the samples was isolated from peripheral blood leucocytes according to the phenol-chloroform method [20]. The extracted DNA was stored at $4{ }^{\circ} \mathrm{C}$ until analysis. Genotyping of the APOA1 rs964184 SNP was performed by polymerase chain reaction and restriction fragment length polymorphism (PCR-RFLP). PCR amplification was performed using 5'-CCATTTCCTTGCAC AACCCA-3' and 5'-ACTGGCCTCTGTATTGACCC-3' as the forward and reversed primer pair, respectively. (Sangon, Shanghai, People's Republic of China). Each amplification reaction was performed in a total volume of $25.0 \mu \mathrm{L}$, including $12.5 \mu \mathrm{L}$ of $2 \times$ Taq PCR MasterMix (constituent: $0.1 \mathrm{U} \mathrm{Taq}$ polymerase $/ \mu \mathrm{L}, 500.0 \mu \mathrm{M}$ dNTP each and PCR buffer), DNase/RNase-free water $\left(\mathrm{ddH}_{2} \mathrm{O}\right)$ $8.5 \mu \mathrm{L}, 1.0 \mu \mathrm{L}$ each primer $(10 \mathrm{pmol} / \mathrm{L})$ and $2.0 \mu \mathrm{L}$ genomic DNA, processing started with $5 \mathrm{~min}$ of predenaturing at $95^{\circ} \mathrm{C}$ and followed by $30 \mathrm{~s}$ of denaturing at $95^{\circ} \mathrm{C}, 30 \mathrm{~s}$ of annealing at $59^{\circ} \mathrm{C}$ and $40 \mathrm{~s}$ of elongation at $72{ }^{\circ} \mathrm{C}$ for 33 cycles. The amplification was completed by a final extension at $72{ }^{\circ} \mathrm{C}$ for $7 \mathrm{~min}$. Following electrophoresis on a $2.0 \%$ agarose gel with $0.5 \mu \mathrm{g} / \mathrm{mL}$ ethidium bromide, the amplification products were visualized under ultraviolet light. The restriction enzyme reaction consisted of $5.0 \mu \mathrm{L}$ amplified DNA, $8.8 \mu \mathrm{L} \mathrm{ddH}_{2} \mathrm{O}, 1.0 \mu \mathrm{L}$ of $10 \times$ buffer solution and $0.2 \mu \mathrm{L} M b o \mathrm{I}$ restriction enzyme [New England Biolabs (Beijing) Ltd.] in a total volume of $15 \mu \mathrm{L}$, which digested at $37^{\circ} \mathrm{C}$ for $40 \mathrm{~min}$. After restriction enzyme digestion of the amplified DNA, genotypes were identified by electrophoresis on $2 \%$ ethidium bromide 
stained agarose gels and visualized under ultraviolet light. Nine samples (each genotype in three; respectively) detected by the PCR-RFLP were also confirmed by direct sequencing. The DNA sequences were analyzed using an ABI Prism 3100 (Applied Biosystems) in Shanghai Sangon Biological Engineering Technology \& Services Co., Ltd., People's Republic of China.

\section{Diagnostic criteria}

The normal values of serum TC, TG, HDL-C, LDL-C, ApoA1, ApoB levels and the ApoA1/ApoB ratio in our Clinical Science Experiment Center were 3.10-5.17, 0.56-1. 70, $1.16-1.42,2.70-3.10 \mathrm{mmol} / \mathrm{L}, 1.20-1.60,0.80-1.05 \mathrm{~g} / \mathrm{L}$ and 1.00-2.50, respectively. The individuals with $\mathrm{TC}>5$. $17 \mathrm{mmol} / \mathrm{L}$ and/or TG $>1.70 \mathrm{mmol} / \mathrm{L}$ were defined as hyperlipidemic [20, 21]. Hypertension was diagnosed according to the 1999 and 2003 criteria of the World Health Organization-International Society of Hypertension Guidelines for the management of hypertension [22]. The diagnostic criteria of overweight and obesity were according to the Cooperative Meta-Analysis Group of China Obesity Task Force. Normal weight, overweight and obesity were defined as a $\mathrm{BMI}<24,24-28$ and $>$ $28 \mathrm{~kg} / \mathrm{m}^{2}$, respectively [23].

\section{Statistical analyses}

The statistical analyses were performed with the statistical software package SPSS 21.0 (SPSS Inc., Chicago, Illinois). The quantitative variables were presented as mean \pm standard deviation (serum TG levels were presented as medians and interquartile ranges). Allele frequency was determined via direct counting, and the Hardy-Weinberg equilibrium was verified with the standard goodness-of-fit test. The genotype distribution between the two groups was analyzed by the chi-square test. General characteristics between two ethnic groups were compared by the Student's unpaired $t$-test. The association between genotypes and serum lipid parameters was tested by covariance analysis (ANCOVA) with gender, age, BMI, blood pressure, alcohol consumption and cigarette smoking as covariates. Multivariable linear regression analyses with stepwise modeling were used to determine the correlation between the genotypes $(\mathrm{CC}=$ $1, C G=2$ and $G G=3$ ) and several environmental factors with serum lipid levels in males and females of Maonan and Han populations. Two-sided $P$ value $<0.05$ was considered statistically significant.

\section{Results}

\section{General characteristics and serum lipid profiles}

The general characteristics and serum lipid levels between the Maonan and Han populations are summarized in Table 1. Systolic blood pressure, pulse pressure, serum TG, and ApoA1 levels were higher in Maonan than in
Han $(P<0.05-0.001)$, whereas the percentages of cigarette smoking, the levels of blood glucose, TG and HDL-C were lower in Maonan than in Han $(P<0.001)$. There was no significant difference in the gender ratio, age structure, body height, weight, BMI, waist circumference, the percentages of alcohol consumption, the levels of diastolic blood pressure, TC, LDL-C, ApoB and the ratio of ApoA1 to ApoB between the two ethnic groups ( $P>0.05$ for all).

\section{Results of electrophoresis and genotyping}

After the genomic DNA of the samples was amplified using PCR and visualized with $2 \%$ agarose gel electrophoresis, the products of $320 \mathrm{bp}$ nucleotide sequences were observed in all samples (Fig. 1). The genotypes identified were termed according to the presence $(\mathrm{G}$ allele) or absence ( $C$ allele) of the enzyme restriction sites. Thus, the CC genotype is homozygous for the absence of the site (bands at $320 \mathrm{bp}$ ), the CG genotype is heterozygous for the presence and absence of the site (bands at 320-, 238- and 82- bp) and the GG genotype is homozygous for the presence of the site (bands at $238 \mathrm{bp}$ and 82 bp; Fig. 2). The position of the APOA1 rs964184 SNP and the CC, CG and GG genotypes confirmed by direct sequencing are shown in Fig. 3, respectively.

\section{Genotypic and allelic frequencies}

The genotypic and allelic frequencies of the APOA1 rs964184 SNP are shown in Table 2. The genotype distribution of the Maonan and Han populations was consistent with the Hardy-Weinberg equilibrium (HWE, $P>0.05)$ by the chi-square test of the goodness of fit. The frequencies of $\mathrm{C}$ and $\mathrm{G}$ alleles were 83.45 and 16 . $55 \%$ in Maonan, and 79.21 and $20.79 \%$ in Han populations $(P<0.05)$, respectively. The frequencies of CC, CG, and GG genotypes were 68.86, 29.18 and $1.96 \%$ in the Maonan population, and $63.78,30.85$ and $5.37 \%$ in the Han population $(P<0.05)$, respectively. No difference in the genotypic and allelic frequencies was found between males and females in each ethnic group $(P>0.05$ for all).

\section{Genotypes and serum lipid levels}

Tables 3 and 4 show the association between genotypes and serum lipid levels. Serum levels of HDL-C were different between the genotypes $(P<0.05)$ in Maonan but not in Han, the $G$ allele carriers had lower serum HDL-C level than the G allele non-carriers. Subgroup analyses showed that the $G$ allele carriers had lower serum HDL-C level in both Maonan males and females $(P<0.05)$. Serum TG levels in Han were different between the genotypes $(P<0.05)$, the $\mathrm{G}$ allele carriers had higher serum levels of TG than the G noncarriers. Subgroup analyses showed that there was no significant difference between Han males and females, but serum ApoA1 levels and the ApoA1/ApoB ratio 
Table 1 Comparison of demographic, lifestyle characteristics and serum lipid levels between the Maonan and Han populations

\begin{tabular}{|c|c|c|c|c|}
\hline Parameter & Maonan & Han & $t\left(x^{2}\right)$ & $P$ \\
\hline Number & 867 & 820 & & \\
\hline Gender (Male/female) & $329 / 538$ & $306 / 514$ & 0.071 & 0.790 \\
\hline Age (year) & $57.71 \pm 14.74$ & $56.34 \pm 15.63$ & 0.672 & 0.513 \\
\hline Height (cm) & $152.90 \pm 11.90$ & $153.99 \pm 7.71$ & -1.425 & 0.154 \\
\hline Weight (kg) & $52.50 \pm 10.96$ & $53.86 \pm 9.20$ & -1.936 & 0.053 \\
\hline Body mass index $\left(\mathrm{kg} / \mathrm{m}^{2}\right)$ & $22.21 \pm 3.91$ & $22.65 \pm 3.11$ & -1.712 & 0.087 \\
\hline Waist circumference $(\mathrm{cm})$ & $75.86 \pm 10.22$ & $75.40 \pm 7.89$ & 0.730 & 0.466 \\
\hline \multicolumn{5}{|l|}{ Cigarette smoking [n(\%)] } \\
\hline Non-smoker & 698(80.46) & $617(75.25)$ & 7.019 & 0.030 \\
\hline$\leq 20$ cigarettes per day & 148(17.08) & $181(22.04)$ & & \\
\hline$>20$ cigarettes per day & $21(2.46)$ & $22(2.71)$ & & \\
\hline \multicolumn{5}{|l|}{ Alcohol consumption [n (\%)] } \\
\hline Non-drinker & $707(81.60)$ & $662(80.68)$ & 2.351 & 0.309 \\
\hline$\leq 25$ g per day & $80(9.20)$ & $92(11.19)$ & & \\
\hline$>25$ g per day & $80(9.20)$ & $66(8.13)$ & & \\
\hline Systolic blood pressure (mmHg) & $135.73 \pm 26.01$ & $130.46 \pm 19.72$ & 3.353 & 0.001 \\
\hline Diastolic blood pressure $(\mathrm{mmHg})$ & $83.57 \pm 13.61$ & $82.52 \pm 11.61$ & 1.144 & 0.253 \\
\hline Pulse pressure $(\mathrm{mmHg})$ & $52.15 \pm 17.91$ & $47.95 \pm 15.47$ & 3.611 & 0.000 \\
\hline Glucose (mmol/L) & $5.69 \pm 2.03$ & $5.97 \pm 1.61$ & -2.045 & 0.041 \\
\hline Total cholesterol (mmol/L) & $4.97 \pm 1.08$ & $4.97 \pm 1.03$ & 0.058 & 0.953 \\
\hline Triglyceride $(\mathrm{mmol} / \mathrm{L})$ & $1.22(0.85)$ & $1.1(0.88)$ & -2.632 & 0.009 \\
\hline $\mathrm{HDL}-\mathrm{C}(\mathrm{mmol} / \mathrm{L})$ & $1.62 \pm 0.40$ & $1.69 \pm 0.42$ & -2.286 & 0.023 \\
\hline $\mathrm{LDL}-\mathrm{C}(\mathrm{mmol} / \mathrm{L})$ & $2.83 \pm 0.81$ & $2.88 \pm 0.87$ & -0.878 & 0.380 \\
\hline Apolipoprotein (Apo) A1 (g/L) & $1.39 \pm 0.32$ & $1.30 \pm 0.25$ & 4.219 & 0.000 \\
\hline ApoB (g/L) & $0.87 \pm 0.20$ & $0.85 \pm 0.20$ & 1.603 & 0.109 \\
\hline ApoA1/ApoB & $1.66 \pm 0.61$ & $1.61 \pm 0.50$ & 1.208 & 0.227 \\
\hline
\end{tabular}

HDL-C high-density lipoprotein cholesterol, $L D L-C$ low-density lipoprotein cholesterol. The value of triglyceride was presented as median (interquartile range), the difference between the two ethnic groups was determined by the Wilcoxon-Mann-Whitney test

were significant different in Han males, the G allele carriers had lower ApoA1 levels and lower ApoA1/ApoB ratio than the $G$ allele non-carriers. No significant difference in the remaining lipid parameters was found between the genotypes in the both populations or in the males and females of the two ethnic groups.

\section{Relative factors for serum lipid parameters}

Multiple linear regression analysis showed that serum HDL-C and ApoA1 levels in the two populations were correlated with the genotypes of APOA1 rs964184 SNP; likewise, serum HDL-C levels in Maonan and TG levels in Han were correlated with the genotypes of the APOA1 rs964184 SNP $(P<0.05$ for each; Table 5$)$. When the correlation of serum lipid parameters and the genotypes was analyzed according to two genders, we found that serum HDL-C levels in Maonan males, ApoA1 levels, and the ApoA1/ApoB ratio in Han males, together with serum TG levels in Han females were all correlated with the genotypes $(P<0.05$; Table 6$)$. Serum lipid parameters were also associated with age, gender, BMI, waist circumference, systolic and diastolic blood pressure, pulse pressure, fasting blood glucose, cigarette smoking and alcohol consumption in both ethnic groups or in males and females $(P<0.05-0.001$; Tables 5 and 6).

\section{Discussion}

The results of our study showed that the serum lipid profiles were different between the Maonan and Han populations. Serum TG and ApoA1 levels were higher in Maonan than in Han $(P<0.05-0.001)$, whereas the levels of serum HDL-C were lower in Maonan than in Han $(P$ $<0.001)$. There was no significant difference in the levels of serum TC, LDL-C, ApoB and the ratio of ApoA1 to ApoB between the two ethnic groups $(P>0.05$ for all). It was widely realized that dyslipidemia as a serious risk 


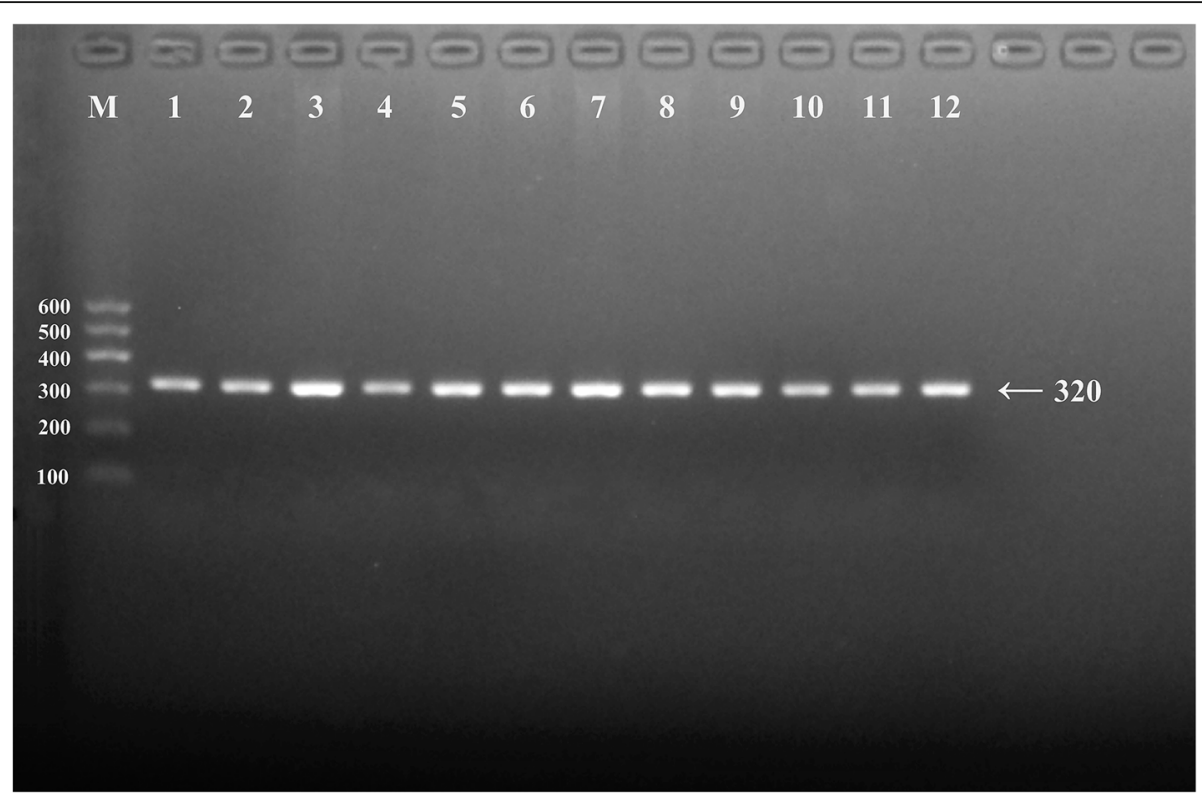

Fig. 1 Electrophoresis of polymerase chain reaction products of the samples. Lane M is the $100 \mathrm{bp}-600 \mathrm{bp}$ marker ladder; Lanes 1-12 are samples, the $320 \mathrm{bp}$ bands are the target genes

factor for CAD is caused by various elements, mainly including genetic and environmental factors and their interaction $[24,25]$. Maonan nationality belongs to a mountain ethnic minority and is mainly occupied with cereal and miscellaneous grain crops. The history of Maonan can retrospect to the eleventh century.
According to the statistics in 2000, the numbers of Maonan population were 107,166 , mainly engaged in agriculture and were good at raising beef cattle and prepare the bamboo hat. The main food for them was rice, along with corn, sorghum, millet, sweet potatoes and pumpkin which are also important complements. Thus, they

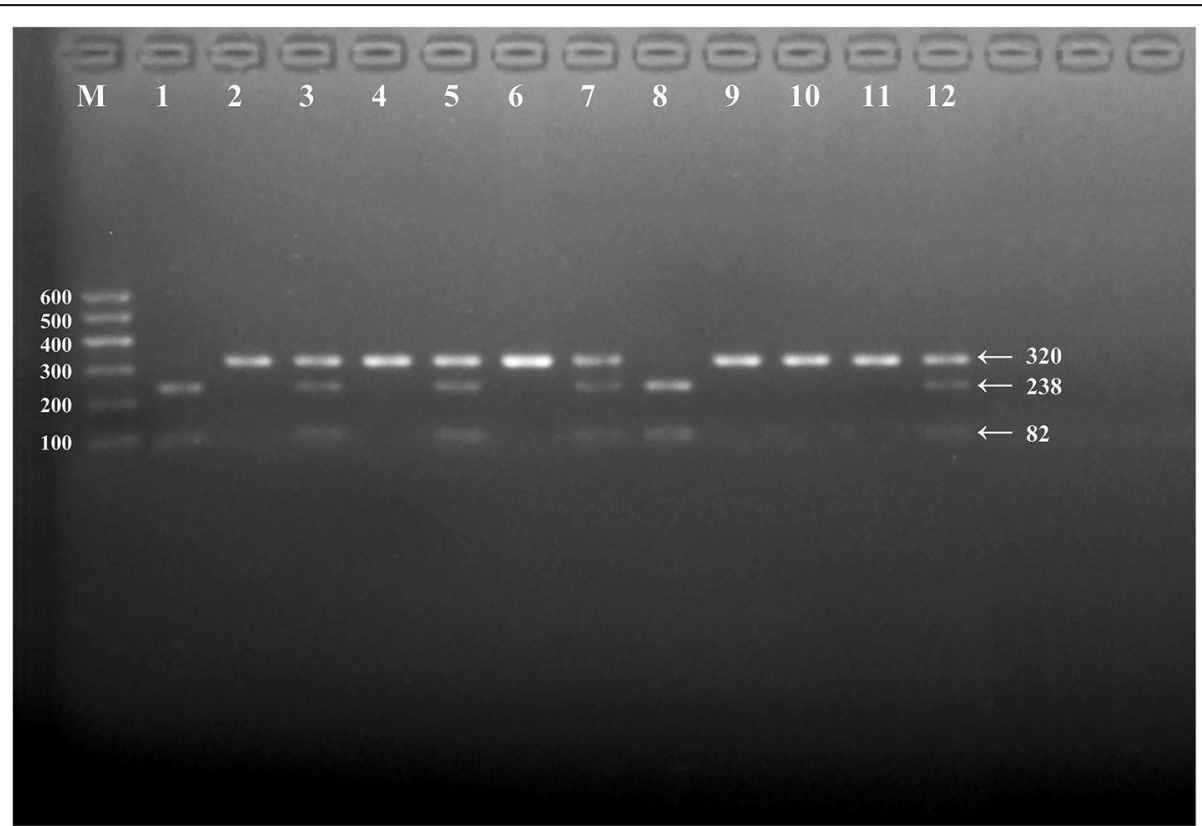

Fig. 2 Genotyping of the APOA1 rs964184 SNP. Lane M is the 100 bp-600 bp marker ladder. Lanes 2, 4, 6, 9, 10 and 11 are the CC genotype (320 bp); lanes 3, 5, 7 and 12 are the CG genotype (320-, 238- and 82- bp); lanes 1 and 8 are the GG genotype (238- and 82- bp) 


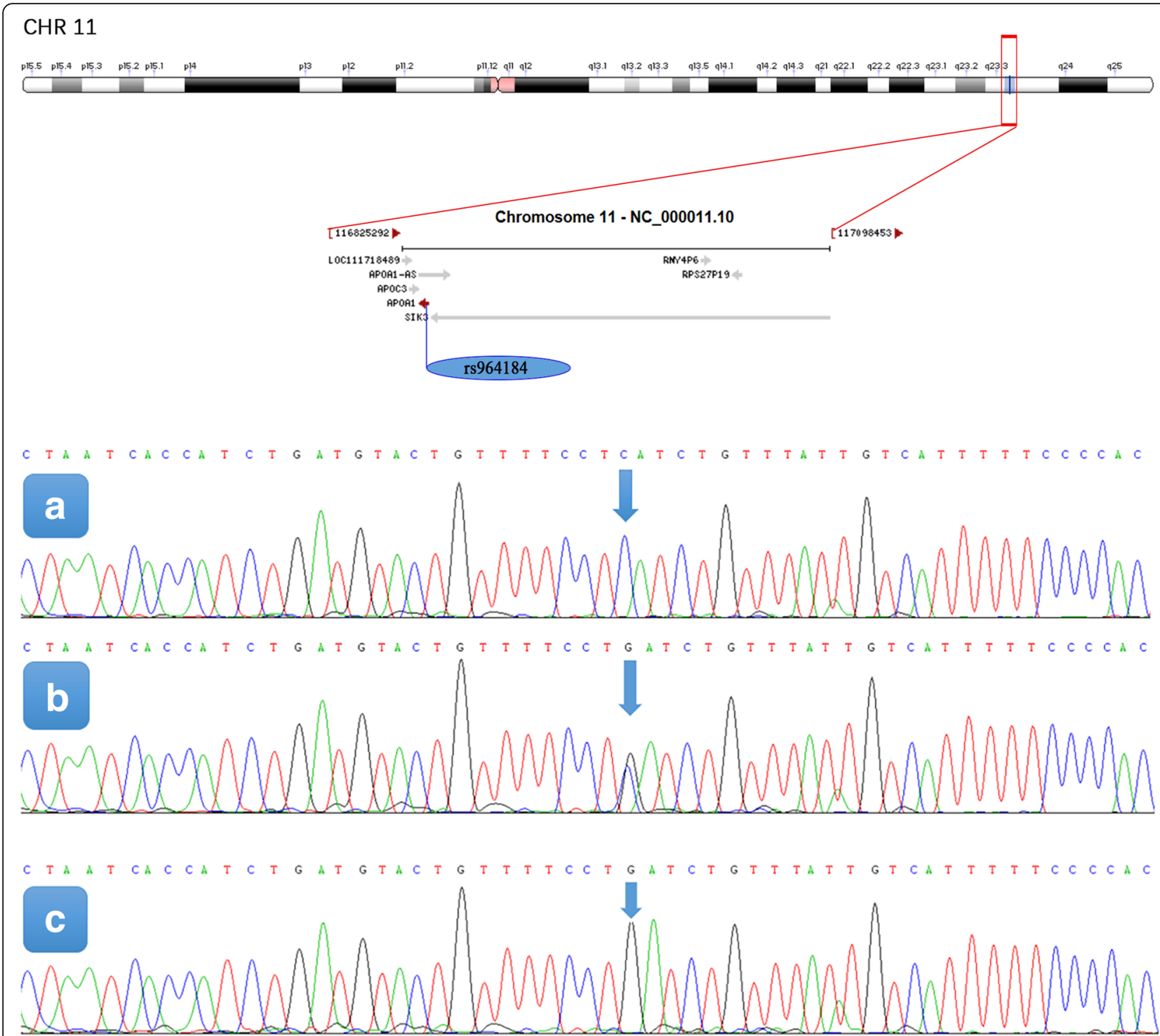

Fig. 3 The position of the APOA1 rs964184 SNP and a part of the nucleotides sequences of the APOA1 rs964184 SNP. A, CC genotype; B, CG genotype; C, GG genotype

enjoyed a very special lifestyle and dietary habits compared with the other nationalities. Maonan people were fond of spicy and acid food. Parents usually were in charge of their children's marriages. Maonan stays endogamy; intermarriage with Han or Zhuang people is seldom happened. Therefore, it is considered that the hereditary characteristics and genotypes of certain lipid metabolism-related genes in this population might be different from those in the Han population.

According to the International HapMap Project's database, the frequencies of $\mathrm{G}$ allele and CG, GG genotypes were 20.73, 31.71 and $4.88 \%$ in Chinese Han in Beijing; 12.05, 22.32 and $0.89 \%$ in European; and 33.14, 52.33 and $6.98 \%$ in Japanese, respectively. While the genotypic and allelic frequencies of the APOA1 rs964184 SNP have not been reported previously in different ethnic groups. In the present study, we firstly showed that the G allele frequency of the APOA1 rs964184 SNP was lower in Maonan than in Han populations $(16.55 \%$ vs. $20.79 \%, P<0.01)$. The distribution of the genotypes was also significantly different between the two ethnic groups $(P<0.001)$, the frequencies of CG and GG genotypes were lower in Maonan than in Han groups, respectively. No significant difference was observed in the genotypic and allelic frequencies between males and females in the two ethnic groups. These results indicate that the prevalence of $A P O A 1$ rs964184 SNP may have $\mathrm{racial} / \mathrm{ethnic}$ specificity. 
Table 2 Comparison of the genotype and allele frequencies of the APOA1 rs964184 SNP in the Maonan and Han populations [n (\%)]

\begin{tabular}{|c|c|c|c|c|c|c|c|}
\hline \multirow[t]{2}{*}{ Group } & \multirow[t]{2}{*}{$n$} & \multicolumn{3}{|l|}{ Genotype } & \multicolumn{2}{|l|}{ Allele } & \multirow[t]{2}{*}{$P_{\text {HWE }}$} \\
\hline & & $\overline{C C}$ & $C G$ & GG & $\bar{C}$ & $G$ & \\
\hline Maonan & 867 & $597(68.86)$ & 253(29.18) & $17(1.96)$ & $1447(83.45)$ & $287(16.55)$ & 0.097 \\
\hline Han & 820 & $523(63.78)$ & 253(30.85) & $44(5.37)$ & 1299(79.21) & $341(20.79)$ & 0.070 \\
\hline$x^{2}$ & & 15.470 & & & 10.009 & & \\
\hline P & & 0.000 & & & 0.002 & & \\
\hline \multicolumn{8}{|l|}{ Maonan } \\
\hline Male & 329 & $231(70.21)$ & $94(28.57)$ & $4(1.22)$ & $556(84.50)$ & $102(15.50)$ & 0.100 \\
\hline Female & 538 & $366(68.03)$ & $159(29.55)$ & $13(2.42)$ & $891(82.81)$ & 185(17.19) & 0.379 \\
\hline$x^{2}$ & & 1.710 & & & 0.846 & & \\
\hline P & & 0.425 & & & 0.358 & & \\
\hline \multicolumn{8}{|l|}{ Han } \\
\hline Male & 306 & 206(67.32) & $87(28.43)$ & $13(4.58)$ & 499(81.37) & $113(18.63)$ & 0.330 \\
\hline Female & 514 & $317(61.67)$ & $166(32.30)$ & $31(6.03)$ & $800(79.28)$ & $228(20.72)$ & 0.144 \\
\hline$x^{2}$ & & 3.024 & & & 3.215 & & \\
\hline$P$ & & 0.221 & & & 0.073 & & \\
\hline
\end{tabular}

HWE Hardy-Weinberg equilibrium. The genotype distribution between the two groups was analyzed by the chi-square test. The HWE was analyzed by the chi-square test of the goodness of fit

To the best of our knowledge, the potential association of the APOA1 rs964184 SNP and serum lipid levels has not been previously reported in different racial/ethnic groups. A previous associated study indicated that the APOA1 encodes a protein, ApoA1, in human beings [12]. ApoA1 could be a better biomarker of cardiovascular risk [10], and it was associated with HDL-C, in another word, it was the most abundant component of HDL-C [14], which played an essential role in formation, maturation and metabolism of HDL-C [15]. Another study about Brazilian people showed that this protein influenced lipid levels and may be risk factors for CVD in the Brazilian elderly [26]. Recently, another GWAS has also identified that genetic variant of the APOA1 rs964184 SNP was associated with serum TG levels mainly, and with TC, HDL-C, LDL-C, secondly [16]. In the current study, we firstly showed that the $\mathrm{G}$ allele carriers in Maonan had lower serum HDL-C levels than the $\mathrm{G}$ allele non-carriers; the $\mathrm{G}$ allele carriers in Han had lower serum TG levels than the G allele noncarriers. Subgroup analyses showed that the G allele carriers in both Maonan males and females had lower serum HDL-C levels, and the G allele carriers in Han males had lower serum ApoA1 levels and the ApoA1/ApoB ratio than the $\mathrm{G}$ allele non-carriers. These findings suggest that there may be an ethnic- and gender-specific association of the APOA1 rs964184 SNP and serum lipid levels.

Table 3 Comparison of the genotypes and serum lipid levels in the Maonan and Han populations

\begin{tabular}{|c|c|c|c|c|c|c|c|c|}
\hline Group/Genotype & $n$ & $\mathrm{TC}(\mathrm{mmol} / \mathrm{L})$ & $\mathrm{TG}(\mathrm{mmol} / \mathrm{L})$ & $\mathrm{HDL}-\mathrm{C}(\mathrm{mmol} / \mathrm{L})$ & LDL-C (mmol/L) & ApoA1 (g/L) & $\mathrm{ApoB}(\mathrm{g} / \mathrm{L})$ & ApoA1/ ApoB \\
\hline \multicolumn{9}{|l|}{ Maonan } \\
\hline $\mathrm{CC}$ & 597 & $5.01 \pm 0.05$ & $1.21(0.80)$ & $1.65 \pm 0.02$ & $2.85 \pm 0.04$ & $1.40 \pm 0.02$ & $0.87 \pm 0.01$ & $1.68 \pm 0.03$ \\
\hline $\mathrm{CG} / \mathrm{GG}$ & 270 & $4.90 \pm 0.08$ & $1.34(1.04)$ & $1.55 \pm 0.03$ & $2.78 \pm 0.06$ & $1.36 \pm 0.02$ & $0.87 \pm 0.01$ & $1.63 \pm 0.04$ \\
\hline$F$ & & 1.138 & -1.506 & 8.790 & 1.063 & 2.131 & 0.010 & 0.818 \\
\hline$P$ & & 0.286 & 0.132 & 0.003 & 0.303 & 0.145 & 0.920 & 0.366 \\
\hline \multicolumn{9}{|l|}{ Han } \\
\hline$C C$ & 523 & $4.97 \pm 0.07$ & $1.04(0.86)$ & $1.71 \pm 0.03$ & $2.90 \pm 0.06$ & $1.31 \pm 0.02$ & $0.85 \pm 0.01$ & $1.63 \pm 0.04$ \\
\hline $\mathrm{CG} / \mathrm{GG}$ & 297 & $4.96 \pm 0.10$ & $1.23(1.16)$ & $1.65 \pm 0.04$ & $2.84 \pm 0.09$ & $1.28 \pm 0.02$ & $0.86 \pm 0.02$ & $1.58 \pm 0.05$ \\
\hline$F$ & & 0.002 & -2.195 & 1.605 & 0.339 & 0.940 & 0.330 & 0.516 \\
\hline$P$ & & 0.962 & 0.028 & 0.206 & 0.561 & 0.333 & 0.566 & 0.473 \\
\hline
\end{tabular}

TC total cholesterol, TG triglyceride, HDL-C high-density lipoprotein cholesterol, $L D L-C$ low-density lipoprotein cholesterol, $A p o A 1$ apolipoprotein A1, $A p o B$ apolipoprotein $\mathrm{B}, A p o A 1 / A p o B$ the ratio of apolipoprotein $\mathrm{A} 1$ to apolipoprotein $\mathrm{B}$. The value of TG was presented as median (interquartile range), the difference between the genotypes was determined by the Wilcoxon-Mann-Whitney test 
Table 4 Comparison of the genotypes and serum lipid levels between males and females in the Maonan and Han populations

\begin{tabular}{|c|c|c|c|c|c|c|c|c|}
\hline Ethnic/Genotype & $\mathrm{n}$ & $\mathrm{TC}(\mathrm{mmol} / \mathrm{L})$ & $\mathrm{TG}(\mathrm{mmol} / \mathrm{L})$ & $\mathrm{HDL}-\mathrm{C}(\mathrm{mmol} / \mathrm{L})$ & $\mathrm{LDL}-\mathrm{C}(\mathrm{mmol} / \mathrm{L})$ & ApoA1 (g/L) & ApoB (g/L) & ApoA1/ApoB \\
\hline \multicolumn{9}{|l|}{ Maonan/Male } \\
\hline $\mathrm{CC}$ & 230 & $4.92 \pm 0.07$ & $1.26(0.87)$ & $1.59 \pm 0.03$ & $2.76 \pm 0.06$ & $1.40 \pm 0.04$ & $0.87 \pm 0.02$ & $1.69 \pm 0.06$ \\
\hline $\mathrm{CG} / \mathrm{GG}$ & 99 & $4.85 \pm 0.11$ & $1.43(1.44)$ & $1.45 \pm 0.05$ & $2.69 \pm 0.09$ & $1.33 \pm 0.05$ & $0.89 \pm 0.02$ & $1.54 \pm 0.09$ \\
\hline$F$ & & 0.247 & -0.837 & 6.996 & 0.317 & 1.452 & 0.412 & 1.784 \\
\hline$P$ & & 0.620 & 0.403 & 0.009 & 0.574 & 0.230 & 0.521 & 0.183 \\
\hline \multicolumn{9}{|l|}{ Maonan/Female } \\
\hline CC & 366 & $5.06 \pm 0.08$ & $1.19(0.68)$ & $1.69 \pm 0.02$ & $2.91 \pm 0.05$ & $1.41 \pm 0.01$ & $0.88 \pm 0.01$ & $1.67 \pm 0.03$ \\
\hline $\mathrm{CG} / \mathrm{GG}$ & 172 & $4.93 \pm 0.11$ & $1.31(0.88)$ & $1.61 \pm 0.04$ & $2.84 \pm 0.08$ & $1.38 \pm 0.02$ & $0.86 \pm 0.02$ & $1.67 \pm 0.05$ \\
\hline$F$ & & 1.001 & -1.361 & 3.903 & 0.676 & 1.025 & 0.316 & 0.000 \\
\hline P & & 0.318 & 0.174 & 0.049 & 0.412 & 0.312 & 0.574 & 0.998 \\
\hline \multicolumn{9}{|l|}{ Han/Male } \\
\hline $\mathrm{CC}$ & 206 & $5.12 \pm 0.11$ & $1.11(0.95)$ & $1.66 \pm 0.04$ & $2.92 \pm 0.09$ & $1.34 \pm 0.03$ & $0.89 \pm 0.02$ & $1.57 \pm 0.05$ \\
\hline CG/GG & 100 & $4.98 \pm 0.16$ & $1.34(2.26)$ & $1.52 \pm 0.06$ & $2.88 \pm 0.13$ & $1.23 \pm 0.04$ & $0.94 \pm 0.03$ & $1.38 \pm 0.07$ \\
\hline$F$ & & 0.519 & -1.140 & 3.669 & 0.077 & 5.286 & 1.359 & 5.272 \\
\hline$P$ & & 0.473 & 0.254 & 0.058 & 0.782 & 0.023 & 0.246 & 0.024 \\
\hline \multicolumn{9}{|l|}{ Han/Female } \\
\hline CC & 317 & $4.87 \pm 0.10$ & $0.97(0.82)$ & $1.74 \pm 0.04$ & $2.88 \pm 0.08$ & $1.30 \pm 0.02$ & $0.82 \pm 0.02$ & $1.67 \pm 0.05$ \\
\hline $\mathrm{CG} / \mathrm{GG}$ & 197 & $4.97 \pm 0.14$ & $1.15(0.83)$ & $1.72 \pm 0.05$ & $2.83 \pm 0.12$ & $1.31 \pm 0.03$ & $0.82 \pm 0.03$ & $1.69 \pm 0.07$ \\
\hline$F$ & & 0.328 & -1.902 & 0.127 & 0.128 & 0.096 & 0.015 & 0.032 \\
\hline P & & 0.567 & 0.057 & 0.722 & 0.721 & 0.758 & 0.901 & 0.858 \\
\hline
\end{tabular}

TC total cholesterol, TG triglyceride, HDL-C high-density lipoprotein cholesterol, LDL-C low-density lipoprotein cholesterol, ApoA1 apolipoprotein A1, ApoB apolipoprotein $B, A p o A 1 / A p o B$ the ratio of apolipoprotein $A 1$ to apolipoprotein $B$. The value of triglyceride was presented as median (interquartile range), the difference among the genotypes was determined by the Wilcoxon-Mann-Whitney test

Previous studies showed that environmental factors such as dietary patterns, lifestyle and physical inactivity are strongly related with serum lipid levels [27]. In the present study, multivariate linear regression analysis also showed that serum lipid parameters were correlated to age, gender, waist circumference, BMI, blood pressure, blood glucose, alcohol consumption, and cigarette smoking in both ethnic groups. These findings suggest that the environmental factors also play an important role in determining or altering serum lipid levels in our study populations. The dietary habits are different between the Maonan and Han populations. Rice is the Maonan people's staple food supplemented with corn, sweet potato and other grains. Maonan people are fond of very strong flavor food, such as eating spicy and acid food with lots of oil and salt. This preference of high in carbohydrates may be related to the higher blood glucose levels, weight, BMI and waist circumference in Maonan than in Han people. In the meantime, rich oil and salt can give rise to higher blood pressure, serum TC, LDL-C and ApoB levels in Maonan than in Han people. Many previous studies proved that diet alone could account for the variability on serum lipid levels [28, 29].
In addition, we also observed that the percentage of cigarette smoking was lower in Maonan than in Han $(P$ $<0.05)$. In multiple linear regression analysis, we could find that alcohol consumption and cigarette smoking may influence serum TC, TG, HDL-C, ApoA1 levels and the ApoA1/ApoB ratio $(P<0.05)$. Several casecontrol and cohort studies have described a J- or Ushaped association between alcohol intake and atherogenesis [30]. A moderate intake of alcohol when taken on a regular amount has been showed to protect against $C A D$ death, which has been attributed to the alterations in serum HDL-C, TG and ApoA1 levels [31]. However, alcohol consumption was also associated with worse hematological values of TC and LDL-C levels. Another research indicated that the effects of alcohol consumption on LDL-C appear to vary by specific patient types or patterns of alcohol intake, and gender as well as genetic variants [32]. In terms of cigarette smoking, one of previous study described that cigarette smoking could be a primary factor in potential changes in lipid profile, which may give rise to the onset of atherosclerosis and CAD in the future [33]. Another study showed that there was difference between two cigarette smoking habits: the length period of smoking and a 
Table 5 Relationship between serum lipid parameters and relative factors in the Maonan and Han populations

\begin{tabular}{|c|c|c|c|c|c|c|}
\hline Lipid & Risk factor & $B$ & Std. error & Beta & $t$ & $P$ \\
\hline \multicolumn{7}{|l|}{ Maonan and Han } \\
\hline \multirow[t]{3}{*}{ TC } & Age & 0.010 & 0.003 & 0.146 & 3.679 & 0.000 \\
\hline & Height & -0.014 & 0.007 & -0.124 & -2.033 & 0.042 \\
\hline & Waist circumference & 0.024 & 0.006 & 0.204 & 3.911 & 0.000 \\
\hline \multirow[t]{4}{*}{ TG } & Alcohol consumption & 0.006 & 0.002 & 0.114 & 3.063 & 0.002 \\
\hline & Height & -0.025 & 0.010 & -0.149 & -2.506 & 0.012 \\
\hline & Weight & 0.047 & 0.018 & 0.300 & 2.581 & 0.010 \\
\hline & Waist circumference & 0.039 & 0.009 & 0.223 & 4.396 & 0.000 \\
\hline \multirow[t]{6}{*}{$\mathrm{HDL}-\mathrm{C}$} & Gender & 0.161 & 0.039 & 0.191 & 4.162 & 0.000 \\
\hline & Alcohol consumption & 0.002 & 0.000 & 0.183 & 4.935 & 0.000 \\
\hline & Waist circumference & -0.009 & 0.002 & -0.212 & -4.198 & 0.000 \\
\hline & Pulse pressure & -0.002 & 0.001 & -0.074 & -2.043 & 0.041 \\
\hline & Ethnic group & 0.070 & 0.028 & 0.082 & 2.497 & 0.013 \\
\hline & Genotype & -0.051 & 0.014 & -0.115 & -3.591 & 0.000 \\
\hline \multirow[t]{3}{*}{ LDL-C } & Age & 0.009 & 0.002 & 0.168 & 4.305 & 0.000 \\
\hline & Alcohol consumption & -0.003 & 0.001 & -0.120 & -3.163 & 0.001 \\
\hline & Waist circumference & 0.019 & 0.005 & 0.211 & 4.135 & 0.000 \\
\hline \multirow[t]{6}{*}{ ApoA1 } & Gender & 0.089 & 0.030 & 0.143 & 3.025 & 0.003 \\
\hline & Cigarette smoking & 0.004 & 0.002 & 0.103 & 2.558 & 0.011 \\
\hline & Alcohol consumption & 0.002 & 0.000 & 0.174 & 4.569 & 0.000 \\
\hline & Waist circumference & -0.004 & 0.002 & -0.108 & -2.083 & 0.038 \\
\hline & Ethnic group & -0.091 & 0.022 & -0.141 & -4.204 & 0.000 \\
\hline & Genotype & -0.023 & 0.011 & -0.069 & -2.103 & 0.036 \\
\hline \multirow[t]{3}{*}{ ApoB } & Age & 0.002 & 0.000 & 0.148 & 3.878 & 0.000 \\
\hline & Height & -0.003 & 0.001 & -0.130 & -2.234 & 0.026 \\
\hline & Waist circumference & 0.007 & 0.001 & 0.330 & 6.614 & 0.000 \\
\hline \multirow[t]{6}{*}{ ApoA1/ApoB } & Gender & 0.177 & 0.054 & 0.148 & 3.273 & 0.001 \\
\hline & Age & -0.004 & 0.001 & -0.114 & -2.977 & 0.003 \\
\hline & Cigarette smoking & 0.008 & 0.003 & 0.103 & 2.661 & 0.008 \\
\hline & Alcohol consumption & 0.003 & 0.001 & 0.176 & 4.801 & 0.000 \\
\hline & Waist circumference & -0.018 & 0.003 & -0.288 & -5.773 & 0.000 \\
\hline & Ethnic group & -0.086 & 0.040 & -0.070 & -2.162 & 0.031 \\
\hline \multicolumn{7}{|l|}{ Maonan } \\
\hline \multirow[t]{3}{*}{ TC } & Age & 0.009 & 0.004 & 0.127 & 2.621 & 0.009 \\
\hline & Gender & 0.276 & 0.129 & 0.124 & 2.138 & 0.033 \\
\hline & Waist circumference & 0.024 & 0.007 & 0.218 & 3.389 & 0.001 \\
\hline \multirow[t]{4}{*}{ TG } & Height & -0.023 & 0.011 & -0.134 & -2.006 & 0.045 \\
\hline & Weight & 0.045 & 0.021 & 0.287 & 2.119 & 0.035 \\
\hline & Alcohol consumption & 0.008 & 0.002 & 0.170 & 3.574 & 0.000 \\
\hline & Waist circumference & 0.038 & 0.011 & 0.217 & 3.471 & 0.001 \\
\hline \multirow[t]{4}{*}{$\mathrm{HDL}-\mathrm{C}$} & Gender & 0.166 & 0.046 & 0.200 & 3.623 & 0.000 \\
\hline & Alcohol consumption & 0.002 & 0.001 & 0.209 & 4.470 & 0.000 \\
\hline & Waist circumference & -0.010 & 0.003 & -0.235 & -3.825 & 0.000 \\
\hline & Pulse pressure & -0.002 & 0.001 & -0.096 & -2.129 & 0.034 \\
\hline
\end{tabular}


Table 5 Relationship between serum lipid parameters and relative factors in the Maonan and Han populations (Continued)

\begin{tabular}{|c|c|c|c|c|c|c|}
\hline Lipid & Risk factor & B & Std. error & Beta & $t$ & $P$ \\
\hline & Genotype & -0.055 & 0.017 & -0.124 & -3.179 & 0.002 \\
\hline \multirow[t]{3}{*}{ LDL-C } & Age & 0.008 & 0.003 & 0.148 & 3.161 & 0.002 \\
\hline & Alcohol consumption & -0.004 & 0.001 & -0.183 & -3.858 & 0.000 \\
\hline & Waist circumference & 0.021 & 0.005 & 0.247 & 3.969 & 0.000 \\
\hline \multirow[t]{3}{*}{ ApoA1 } & Gender & 0.092 & 0.039 & 0.138 & 2.392 & 0.017 \\
\hline & Alcohol consumption & 0.001 & 0.000 & 0.149 & 3.051 & 0.002 \\
\hline & Waist circumference & -0.005 & 0.002 & -0.137 & -2.131 & 0.034 \\
\hline \multirow[t]{2}{*}{ ApoB } & Age & 0.002 & 0.001 & 0.159 & 3.470 & 0.001 \\
\hline & Waist circumference & 0.008 & 0.001 & 0.376 & 6.176 & 0.000 \\
\hline \multirow[t]{3}{*}{ ApoA1/ApoB } & Age & -0.005 & 0.002 & -0.131 & -2.852 & 0.005 \\
\hline & Alcohol consumption & 0.003 & 0.001 & 0.183 & 3.955 & 0.000 \\
\hline & Waist circumference & -0.020 & 0.004 & -0.325 & -5.336 & 0.000 \\
\hline \multicolumn{7}{|l|}{ Han } \\
\hline \multirow[t]{2}{*}{ TC } & Age & 0.010 & 0.005 & 0.147 & 2.133 & 0.034 \\
\hline & Diastolic blood pressure & 0.014 & 0.005 & 0.162 & 2.670 & 0.008 \\
\hline \multirow[t]{4}{*}{ TG } & Cigarette smoking & 0.035 & 0.012 & 0.202 & 3.056 & 0.002 \\
\hline & Waist circumference & 0.043 & 0.016 & 0.245 & 2.696 & 0.007 \\
\hline & Diastolic blood pressure & 0.015 & 0.007 & 0.129 & 2.207 & 0.028 \\
\hline & Genotype & 0.248 & 0.083 & 0.164 & 2.993 & 0.003 \\
\hline \multirow[t]{2}{*}{$\mathrm{HDL}-\mathrm{C}$} & Gender & 0.166 & 0.074 & 0.193 & 2.247 & 0.025 \\
\hline & Alcohol consumption & 0.002 & 0.001 & 0.152 & 2.426 & 0.016 \\
\hline \multirow[t]{2}{*}{ LDL-C } & Age & 0.010 & 0.004 & 0.170 & 2.457 & 0.015 \\
\hline & Cigarette smoking & -0.018 & 0.008 & -0.163 & -2.384 & 0.018 \\
\hline \multirow[t]{3}{*}{ ApoA1 } & Gender & 0.097 & 0.045 & 0.187 & 2.172 & 0.031 \\
\hline & Alcohol consumption & 0.002 & 0.001 & 0.263 & 4.190 & 0.000 \\
\hline & Cigarette smoking & 0.006 & 0.002 & 0.173 & 2.497 & 0.013 \\
\hline \multirow[t]{2}{*}{ ApoB } & Gender & -0.099 & 0.034 & -0.236 & -2.881 & 0.004 \\
\hline & Waist circumference & 0.006 & 0.002 & 0.226 & 2.476 & 0.014 \\
\hline \multirow[t]{3}{*}{ ApoA1/ApoB } & Gender & 0.358 & 0.087 & 0.343 & 4.123 & 0.000 \\
\hline & Cigarette smoking & 0.011 & 0.004 & 0.171 & 2.550 & 0.011 \\
\hline & Alcohol consumption & 0.003 & 0.001 & 0.161 & 2.648 & 0.009 \\
\hline
\end{tabular}

$T C$ total cholesterol, TG triglyceride, $H D L-C$ high-density lipoprotein cholesterol, $L D L-C$ low-density lipoprotein cholesterol, $A p o A 1$ apolipoprotein $A 1, A p o B$ apolipoprotein $\mathrm{B}, A p o A 1 / A p o B$ the ratio of apolipoprotein $\mathrm{A} 1$ to apolipoprotein $\mathrm{B}, \mathrm{B}$ unstandardized coefficient, Beta standardized coefficient

number of cigarettes smoked daily, and made a conclusion that more reflection to the status of lipids has the bigger number of smoked cigarettes daily than the length of the period of cigarette smoking [34]. Therefore, the results of exposure to different lifestyle and environmental factors probably further modify the association of genetic variations and serum lipid levels in our study populations.

\section{Limitations}

There are several potential limitations in our study. Firstly, we were not able to alleviate the effect of diet and some environmental factors during the statistical analysis. Secondly, we could not completely exclude asymptomatic disorders, atherosclerosis, for instance, which could create a potentially significant bias due to poor field study condition. Thirdly, there are still many unmeasured environmental and genetic factors which should be considered, although we have observed significant association of the APOA1 rs964184 SNP and serum lipid levels. In addition, the interactions of gene-gene, gene-environment, and environment-environment on serum lipid levels are remained to be confirmed. 
Table 6 Relationship between serum lipid parameters and relative factors in the males and females of the both ethnic groups

\begin{tabular}{|c|c|c|c|c|c|c|}
\hline Lipid & Risk factor & $\mathrm{B}$ & Std. error & Beta & $t$ & $P$ \\
\hline \multicolumn{7}{|l|}{ Maonan/ male } \\
\hline TG & Alcohol consumption & 0.010 & 0.004 & 0.188 & 2.605 & 0.010 \\
\hline \multirow[t]{3}{*}{$\mathrm{HDL}-\mathrm{C}$} & Alcohol consumption & 0.003 & 0.001 & 0.323 & 4.928 & 0.000 \\
\hline & Waist circumference & -0.018 & 0.005 & -0.480 & -3.884 & 0.000 \\
\hline & Genotype & -0.070 & 0.027 & -0.155 & -2.588 & 0.010 \\
\hline LDL-C & Alcohol consumption & -0.004 & 0.001 & -0.274 & -3.851 & 0.000 \\
\hline \multirow[t]{2}{*}{ ApoA1 } & Alcohol consumption & 0.002 & 0.001 & 0.175 & 2.420 & 0.016 \\
\hline & Waist circumference & -0.014 & 0.005 & -0.344 & -2.503 & 0.013 \\
\hline ApoB & Weight & 0.008 & 0.003 & 0.432 & 2.449 & 0.015 \\
\hline \multirow[t]{3}{*}{ ApoA1/ApoB } & Age & -0.009 & 0.004 & -0.174 & -2.256 & 0.025 \\
\hline & Alcohol consumption & 0.003 & 0.001 & 0.212 & 3.080 & 0.002 \\
\hline & Waist circumference & -0.022 & 0.009 & -0.310 & -2.391 & 0.018 \\
\hline \multicolumn{7}{|l|}{ Maonan/ female } \\
\hline \multirow[t]{2}{*}{ TC } & Age & 0.011 & 0.005 & 0.141 & 2.335 & 0.020 \\
\hline & Waist circumference & 0.029 & 0.010 & 0.209 & 2.947 & 0.003 \\
\hline TG & Waist circumference & 0.026 & 0.006 & 0.308 & 4.530 & 0.000 \\
\hline \multirow[t]{2}{*}{ LDL-C } & Age & 0.009 & 0.003 & 0.157 & 2.688 & 0.008 \\
\hline & Waist circumference & 0.029 & 0.007 & 0.297 & 4.336 & 0.000 \\
\hline \multirow[t]{2}{*}{ ApoB } & Age & 0.002 & 0.001 & 0.164 & 2.910 & 0.004 \\
\hline & Waist circumference & 0.010 & 0.002 & 0.406 & 6.135 & 0.000 \\
\hline \multirow[t]{2}{*}{ ApoA1 /ApoB } & Waist circumference & -0.018 & 0.004 & -0.308 & -4.507 & 0.000 \\
\hline & Pulse pressure & -0.004 & 0.002 & -0.131 & -2.336 & 0.020 \\
\hline \multicolumn{7}{|l|}{ Han/male } \\
\hline TG & Cigarette smoking & 0.035 & 0.014 & 0.239 & 2.581 & 0.011 \\
\hline \multirow[t]{2}{*}{$\mathrm{HDL}-\mathrm{C}$} & Alcohol consumption & 0.002 & 0.001 & 0.194 & 2.099 & 0.038 \\
\hline & Diastolic blood pressure & 0.008 & 0.004 & 0.246 & 2.253 & 0.026 \\
\hline LDL-C & Cigarette smoking & -0.018 & 0.008 & -0.234 & -2.381 & 0.019 \\
\hline \multirow[t]{3}{*}{ ApoA1 } & Cigarette smoking & 0.006 & 0.002 & 0.245 & 2.696 & 0.008 \\
\hline & Alcohol consumption & 0.002 & 0.001 & 0.376 & 4.258 & 0.000 \\
\hline & Genotype & -0.054 & 0.026 & -0.185 & -2.074 & 0.041 \\
\hline \multirow[t]{3}{*}{ ApoA1 /ApoB } & Cigarette smoking & 0.012 & 0.004 & 0.271 & 3.012 & 0.003 \\
\hline & Alcohol consumption & 0.003 & 0.001 & 0.258 & 2.952 & 0.004 \\
\hline & Genotype & -0.091 & 0.045 & -0.180 & -2.040 & 0.044 \\
\hline \multicolumn{7}{|l|}{ Han/ female } \\
\hline TC & Age & 0.091 & 0.007 & 0.267 & 2.898 & 0.004 \\
\hline \multirow[t]{2}{*}{ TG } & Diastolic blood pressure & 0.021 & 0.009 & 0.183 & 2.308 & 0.022 \\
\hline & Genotype & 0.311 & 0.098 & 0.229 & 3.163 & 0.002 \\
\hline \multirow[t]{4}{*}{ LDL-C } & Age & 0.023 & 0.005 & 0.380 & 4.254 & 0.000 \\
\hline & Height & 0.176 & 0.073 & 1.156 & 2.426 & 0.016 \\
\hline & Weight & -0.270 & 0.109 & -2.179 & -2.487 & 0.014 \\
\hline & Body mass index & 0.616 & 0.246 & 2.045 & 2.506 & 0.013 \\
\hline ApoB & Body mass index & 0.112 & 0.055 & 1.698 & 2.025 & 0.044 \\
\hline
\end{tabular}

TC total cholesterol, TG triglyceride, HDL-C high-density lipoprotein cholesterol, LDL-C low-density lipoprotein cholesterol, $A p o A 1$ apolipoprotein $A 1, A p o B$ apolipoprotein B, ApoA1/ApoB the ratio of apolipoprotein A1 to apolipoprotein B, B unstandardized coefficient, Beta standardized coefficient. The correlation among serum lipid parameters and the genotypes and several environmental factors was determined by multivariable linear regression analyses with stepwise modeling 


\section{Conclusions}

The present study showed that the genotypic and allelic frequencies of the APOA1 rs964184 SNP were significantly different between the Maonan and Han populations. The associations of the APOA1 rs964184 SNP and serum lipid levels were also significantly different between the two ethnic groups and between males and females in the Maonan population. There may be a racial/ ethnic- and/or gender-specific association of the APOA1 rs964184 SNP and serum lipid levels.

\section{Abbreviations \\ ANCOVA: Analysis of covariance; Apo: Apolipoprotein; APOA1: Apolipoprotein A1; BMI: Body mass index; CAD: Coronary artery disease; CVD: Cardiovascular disease; GWAS: Genome-wide association study; HDL-C: High-density lipoprotein cholesterol; HWE: Hardy-Weinberg equilibrium; LDL-C: Low-density lipoprotein cholesterol; PCR: Polymerase chain reaction; RFLP: Restriction fragment length polymorphism; SNP: Single nucleotide polymorphism; TC: Total cholesterol; TG: Triglyceride}

\section{Funding}

This study was supported by the National Natural Science Foundation of China (No: 81460169).

\section{Availability of data and materials}

The datasets generated during the present study are not publicly available, because detailed genetic information of each participant was included in these materials.

\section{Authors' contributions}

LQ conceived the study, participated in the design, undertook genotyping, performed the statistical analyses, and drafted the manuscript. RXY conceived the study, participated in the design, carried out the epidemiological survey, collected the samples, and helped to draft the manuscript. EK collaborated to the genotyping. FHZ, DZY and SLP carried out the epidemiological survey, and collected the samples. All authors read and approved the final manuscript.

\section{Ethics approval and consent to participate}

All procedures of the investigation were carried out following the rules of the Declaration of Helsinki, seventh revision (2013). The study design was approved by the Ethics Committee of the First Affiliated Hospital, Guangxi Medical University (No: Lunshen-2014-KY-Guoji-001; March 7, 2014). Informed consent was obtained from all participants.

\section{Competing interests}

The authors declare that they have no competing interests.

\section{Publisher's Note}

Springer Nature remains neutral with regard to jurisdictional claims in published maps and institutional affiliations.

\section{Author details \\ ${ }^{1}$ Department of Cardiology, Institute of Cardiovascular Diseases, The First Affiliated Hospital, Guangxi Medical University, 22 Shuangyong Road, Nanning 530021, Guangxi, People's Republic of China. ${ }^{2}$ Department of Molecular Genetics, Medical Scientific Research Center, Guangxi Medical University, Nanning 530021, Guangxi, People's Republic of China. ${ }^{3}$ Department of Pathophysiology, School of Premedical Sciences, Guangxi Medical University, Nanning 530021, Guangxi, People's Republic of China.}

Received: 4 December 2017 Accepted: 24 April 2018

Published online: 10 May 2018

\section{References}

1. Yazdanyar A, Newman AB. The burden of cardiovascular disease in the elderly: morbidity, mortality, and costs. Clin Geriatr Med. 2009;25:563-77.

2. Lozano R, Naghavi M, Foreman K, Lim S, Shibuya K, Aboyans V, Abraham J, Adair T, Aggarwal R, Ahn SY, et al. Global and regional mortality from
235 causes of death for 20 age groups in 1990 and 2010: a systematic analysis for the global burden of disease study 2010. Lancet. 2012;380: 2095-128.

3. D'Agostino RB Sr, Vasan RS, Pencina MJ, Wolf PA, Cobain M, Massaro JM, Kannel WB. General cardiovascular risk profile for use in primary care: the Framingham heart study. Circulation. 2008;117:743-53.

4. Sin HY, Jin YK, Jung KH. Total cholesterol, high density lipoprotein and triglyceride for cardiovascular disease in elderly patients treated with metformin. Arch Pharm Res. 2011;34:99-107.

5. Chapman MJ, Ginsberg HN, Amarenco P, Andreotti F, Borén J, Catapano AL, Descamps OS, Fisher E, Kovanen PT, Kuivenhoven JA. Triglyceride-rich lipoproteins and high-density lipoprotein cholesterol in patients at high risk of cardiovascular disease: evidence and guidance for management. Eur Heart J. 2015;32:1345-61.

6. Okamura T, Kokubo Y, Watanabe M, Higashiyama A, Miyamoto Y, Yoshimasa Y, Okayama A. Low-density lipoprotein cholesterol and non-high-density lipoprotein cholesterol and the incidence of cardiovascular disease in an urban Japanese cohort study: the Suita study. Atherosclerosis. 2009;203: 587-92.

7. Berkinbayev S, Rysuly M, Mussayev A, Blum K, Baitasova N, Mussagaliyeva A, Dzhunusbekova G, Makhatov B, Mussayev AA, Yeshmanova A, et al. Apolipoprotein gene polymorphisms (APOB, APOC111, APOE) in the development of coronary heart disease in ethnic groups of Kazakhstan. J Genet Syndr Gene Ther. 2014;5:216.

8. Walldius $G$, Jungner I. The apoB/apoA-I ratio: a strong, new risk factor for cardiovascular disease and a target for lipid-lowering therapy-a review of the evidence. J Intern Med. 2006;259:493-519.

9. Castelli WP. Cholesterol and lipids in the risk of coronary artery disease-the Framingham heart study. Can J Cardiol. 1988;4(Suppl A):5A-10A.

10. Casillas-Munoz F, Valle Y, Munoz-Valle JF, Martinez-Fernandez DE, ReynosoVillalpando GL, Flores-Salinas HE, Llamas-Covarrubias MA, Padilla-Gutierrez JR. APOA1 and APOB polymorphisms and apolipoprotein concentrations as biomarkers of risk in acute coronary syndrome: relationship with lipidlowering therapy effectiveness. Med Clin (Barc). 2017. https://doi.org/10. 1016/j.medcli.2017.07.026.

11. Lee YC, Lai CQ, Ordovas JM, Parnell LD. A database of gene-environment interactions pertaining to blood lipid traits, cardiovascular disease and type 2 diabetes. J Data Mining Genomics Proteomics. 2011;2:106.

12. Breslow JL, Ross D, McPherson J, Williams H, Kurnit D, Nussbaum AL, Karathanasis SK, Zannis VI. Isolation and characterization of CDNA clones for human apolipoprotein A-I. Proc Natl Acad Sci U S A. 1982;79:6861-5.

13. Arinami T, Hirano T, Kobayashi K, Yamanouchi Y, Hamaguchi H. Assignment of the apolipoprotein A-I gene to 11q23 based on RFLP in a case with a partial deletion of chromosome 11, del(11)(q23.3_- qter). Hum Genet. 1990:85:39-40.

14. Rye KA, Bursill CA, Lambert G, Tabet F, Barter PJ. The metabolism and antiatherogenic properties of HDL. J Lipid Res. 2009;50:S195-200.

15. Bandarian F, Hedayati M, Daneshpour MS, Naseri M, Azizi F. Genetic polymorphisms in the APOA1 gene and their relationship with serum HDL cholesterol levels. Lipids. 2013;48:1207-16.

16. Teslovich TM, Musunuru K, Smith AV, Edmondson AC, Stylianou IM, Koseki M, Pirruccello JP, Ripatti S, Chasman DI, Willer CJ, et al. Biological, clinical and population relevance of 95 loci for blood lipids. Nature. 2010;466:707-13.

17. Wang Y, Aung LH, Tan JY, Yin RX, Hu XJ, Long XJ, Wu DF, Miao L, Yang DZ, Pan SL. Prevalence of dyslipidemia and its risk factors in the Chinese Maonan and Han populations. Int J Clin Exp Pathol. 2016;9:10603-16.

18. An epidemiological study of cardiovascular and cardiopulmonary disease risk factors in four populations in the People's republic of China. Baseline report from the P.R.C.-U.S.a. collaborative study. People's republic of ChinaUnited States cardiovascular and cardiopulmonary epidemiology research group. Circulation. 1992;85:1083-96.

19. Guo T, Yin RX, Lin QZ, Wu J, Shen SW, Sun JQ, Shi GY, Wu JZ, Li H, Wang YM. Polymorphism of rs 873308 near the transmembrane protein 57 gene is associated with serum lipid levels. Biosci Rep. 2014;34:69-81.

20. Zhang QH, Yin RX, Gao H, Huang F, Wu JZ, Pan SL, Lin WX, Yang DZ. Association of the SPTLC3 rs364585 polymorphism and serum lipid profiles in two Chinese ethnic groups. Lipids Health Dis. 2017;16:1.

21. Grönroos P, Raitakari OT, Kähönen M, Hutri-Kähönen N, Juonala M, Marniemi J, Viikari J, Lehtimäki T. Relation of apolipoprotein E polymorphism to markers of early atherosclerotic changes in young adults-the cardiovascular risk in young Finns study. Circ J. 2008;72:29-34. 
22. Whitworth JA. World health organization, International Society of Hypertension Writing Group. 2003 World Health Organization (WHO)/ International Society of Hypertension (ISH) statement on management of hypertension. J Hypertens. 2003;21:1983-92.

23. Zhou B. Coorperative meta-analysis group of China obesity task force. Predictive values of body mass index and waist circumference to risk factors of related diseases in Chinese adult population. Zhonghua Liu Xing Bing Xue Za Zhi. 2002;23:5-10.

24. Zhang L, Yin RX, Liu WY, Miao L, Wu DF, Aung LH, Hu XJ, Cao XL, Wu JZ, Pan SL. Association of methylenetetrahydrofolate reductase C677T polymorphism and serum lipid levels in the Guangxi Bai Ku Yao and Han populations. Lipids Health Dis. 2010;9:123.

25. Martin SS, Blaha MJ, Blankstein R, Agatston A, Rivera JJ, Virani SS, Ouyang P, Jones SR, Blumenthal RS, Budoff MJ, et al. Dyslipidemia, coronary artery calcium, and incident atherosclerotic cardiovascular disease: implications for statin therapy from the multi-ethnic study of atherosclerosis. Circulation. 2014;129:77-86.

26. Furuya TK, Chen ES, Ota VK, Mazzotti DR, Ramos LR, Cendoroglo MS, Araujo $\mathrm{LQ}$, Burbano RR, Smith MAC. Association of APOA1 and APOA5 polymorphisms and haplotypes with lipid parameters in a Brazilian elderly cohort. Genet Mol Res. 2013;12:3495-9.

27. Robinson D, Kawamura T, Hinohara S, Sakamoto Y, Takahashi T. Levels of cardiovascular risk factors in Japanese people living in the UK. J Cardiovasc Risk. 1995:2:449-58.

28. Joffe $Y T$, Collins M, Goedecke $\mathrm{JH}$. The relationship between dietary fatty acids and inflammatory genes on the obese phenotype and serum lipids. Nutrients. 2013;5:1672-705.

29. Erkkila AT, Sarkkinen ES, Lehto S, Pyorala K, Uusitupa MI. Dietary associates of serum total, $\mathrm{LDL}$, and $\mathrm{HDL}$ cholesterol and triglycerides in patients with coronary heart disease. Prev Med. 1999;28:558-65.

30. Rimm EB, Williams P, Fosher K, Criqui M, Stampfer MJ. Moderate alcohol intake and lower risk of coronary heart disease: meta-analysis of effects on lipids and haemostatic factors. BMJ. 1999;319:1523-8.

31. De Oliveira E Silva ER, Foster D, McGee Harper M, Seidman CE, Smith JD, Breslow JL, Brinton EA. Alcohol consumption raises HDL cholesterol levels by increasing the transport rate of apolipoproteins A-I and A-II. Circulation. 2000:102:2347-52.

32. Rees K, Hartley L, Flowers N, Clarke A, Hooper L, Thorogood M, Stranges S. 'Mediterranean' dietary pattern for the primary prevention of cardiovascular disease. Cochrane Database Syst Rev. 2013;(8):CD009825. https://doi.org/10. 1002/14651858.CD009825.

33. Sliwinska-Mosson M, Mihulka E, Milnerowicz H. Assessment of lipid profile in non-smoking and smoking young health persons. Przegl Lek. 2014;71:585-7.

34. Bisanovic S, Mehic B, Sivic S. Status of lipids and the frequency diseases of cardiovascular origin in smokers according to the length period of smoking and a number of cigarettes smoked daily. Bosn J Basic Med Sci. 2011; 11:46-51.

\section{Ready to submit your research? Choose BMC and benefit from:}

- fast, convenient online submission

- thorough peer review by experienced researchers in your field

- rapid publication on acceptance

- support for research data, including large and complex data types

- gold Open Access which fosters wider collaboration and increased citations - maximum visibility for your research: over $100 \mathrm{M}$ website views per year

At BMC, research is always in progress.

Learn more biomedcentral.com/submissions 